\title{
Wavelength Scaling of High Harmonic Generation Efficiency
}

\author{
A. D. Shiner, ${ }^{1}$ C. Trallero-Herrero, ${ }^{1}$ N. Kajumba, ${ }^{1}$ H.-C. Bandulet, ${ }^{2}$ D. Comtois, ${ }^{2}$ F. Légaré, ${ }^{2}$ M. Giguère, ${ }^{2}$ J-C. Kieffer, ${ }^{2}$ \\ P. B. Corkum, ${ }^{1}$ and D. M. Villeneuve ${ }^{1, *}$ \\ ${ }^{1}$ National Research Council of Canada, 100 Sussex Drive, Ottawa, Ontario K1A OR6, Canada \\ ${ }^{2}$ INRS-Énergie et Matériaux, 1650 boulevard Lionel-Boulet, C.P. 1020, Varennes (Québec) J3X 1S2, Canada
}

(Received 16 April 2009; published 14 August 2009)

\begin{abstract}
Using longer wavelength laser drivers for high harmonic generation is desirable because the highest extreme ultraviolet frequency scales as the square of the wavelength. Recent numerical studies predict that high harmonic efficiency falls dramatically with increasing wavelength, with a very unfavorable $\lambda^{-(5-6)}$ scaling. We performed an experimental study of the high harmonic yield over a wavelength range of 800 $1850 \mathrm{~nm}$. A thin gas jet was employed to minimize phase matching effects, and the laser intensity and focal spot size were kept constant as the wavelength was changed. Ion yield was simultaneously measured so that the total number of emitting atoms was known. We found that the scaling at constant laser intensity is $\lambda^{-6.3 \pm 1.1}$ in Xe and $\lambda^{-6.5 \pm 1.1}$ in $\mathrm{Kr}$ over the wavelength range of $800-1850 \mathrm{~nm}$, somewhat worse than the theoretical predictions.
\end{abstract}

DOI: 10.1103/PhysRevLett.103.073902

PACS numbers: 42.65.Ky, 32.80.Wr, 42.65.Re

High harmonic generation (HHG) is a three-step process $[1,2]$ in which an intense laser removes an electron from an atom or molecule, accelerates the electron, and then recombines the electron with the parent ion. The resulting emission extends to the extreme ultraviolet (XUV) and is composed of odd harmonics of the driving laser frequency. HHG provides a source of attosecond pulses [3-5]. The recombination of the recolliding electron also provides a measure of the electronic structure of molecules [6]. Increasing the extent of the XUV spectrum can lead to shorter attosecond pulses [5] and will give better resolution for molecular orbital imaging [6] by reducing the electron's de Broglie wavelength [7].

The highest frequency produced [8], for a given ionization state, is approximately $\Omega_{\max }=3.17 U_{p}+I_{p}$, where $U_{p}=F^{2} /\left(4 \omega^{2}\right)$ is the ponderomotive energy, $F$ is the peak laser electric field, $\omega$ is the laser angular frequency, and $I_{p}$ is the ionization potential of that state (atomic units are used). $U_{p}$ scales as $I \lambda^{2}$, where $I$ is the peak laser intensity and $\lambda$ is the laser wavelength. One approach to extend the cutoff frequency $\Omega_{\max }$ is to increase the laser intensity. Unfortunately, there is a practical limit beyond which the intensity cannot be increased-the point at which the state is fully ionized.

An alternate approach is to use longer laser wavelengths $[9,10]$. The $\lambda^{2}$ scaling of $\Omega_{\max }$ is unfortunately predicted to be offset by an unfavorable scaling of the conversion efficiency from laser energy into XUV energy due to the spreading of the continuum electron wave packet in both space and time. This was first explored numerically by Tate et al. [11] using time-dependent Schrödinger and strong field approximation calculations to show that the singleatom HHG efficiency $\eta(\lambda) \propto \lambda^{-(5-6)}$ at constant intensity when integrated over a few tens of eV in the XUV spectrum. This power law has been verified by further theoretical studies $[12,13]$.
There have been several experimental studies on the dependence of different properties of HHG on laser wavelength. These studies include a demonstration that the cutoff frequency is extended with longer wavelength drivers but with a loss of efficiency [10,14], and measurements of the phase matching conditions that optimize the XUV cutoff for different wavelengths [15-17]. There has also been a recent report of the wavelength dependence of the attosecond chirp [18]. Despite strong interest in the wavelength scaling of HHG efficiency by the attosecond community, there are very few quantitative studies reported in the literature. Shan and Chang [10] compared the intensity of the 37th harmonic in Xe for three wavelengths from 1.22 to $1.51 \mu \mathrm{m}$. They found that from the first to the second wavelength the harmonic intensity decreased as roughly the cube of the free electron transit time as predicted by Lewenstein et al. [8]. The measurement for the third wavelength deviated from this trend by nearly a factor of 2 . Colosimo et al. [19] reported measurements at two wavelengths. Their experiment was not designed to be sensitive to the single atoms response, and the large decrease in HHG efficiency with increasing wavelength was shown to be sensitive to the gas density and hence to phase matching. Thus, experimental confirmation of the wavelength scaling at the single-atom level has remained elusive.

It is difficult to design an experiment in which the laser wavelength is the only parameter that changes. We wish to provide experimental confirmation of the single-atom calculations, and so we must avoid collective effects such as phase mismatch, differing focal spot diameters, and different intensities. We have carefully arranged the experiment so that all of the important parameters are controlled. (1) The laser beam is spatially filtered through a hollow core fiber capillary at all wavelengths. (2) The output of the capillary is relay-imaged into the gas jet in which harmonics are generated, providing the same focal spot diameter at 
all wavelengths. (3) The gas jet is only $0.5 \mathrm{~mm}$ thick, to minimize the possibility of phase mismatch and reabsorption. (4) The number of ions produced is independently measured. (5) The intensity in the gas jet is determined at each wavelength by measuring the ion yield versus intensity. (6) As a final correction, the high harmonic yield is normalized by the ion yield squared so that the emission per atom is measured.

The experimental arrangement is shown in Fig. 1. The $800 \mathrm{~nm}$ radiation was produced directly by a Ti:sapphire laser (Thales, $100 \mathrm{~Hz}, 80 \mathrm{~mJ}, 30 \mathrm{fs}$ ). Longer wavelengths were produced by directing $7 \mathrm{~mJ}$ of the $800 \mathrm{~nm}$ radiation into an optical parametric amplifier (OPA) (Light Conversion TOPAS-HE) which output $2.3 \mathrm{~mJ}$ (signal + idler). From this source we selected three wavelengths: 1300, 1400 (signal), and $1850 \mathrm{~nm}$ (idler). Further details can be found in Ref. [20].

Each source was coupled into a $400 \mu \mathrm{m}$-diameter hollow core fiber which performs spatial filtering and provides a well-defined spatial mode for harmonic generation regardless of wavelength [20]. The mode exiting the fiber was imaged into the gas jet with the $4 f$ image relay geometry shown in Fig. 1. The distance between the collimating and focusing lenses was equal to the sum of the focal lengths of each lens. The $4 f$ geometry ensures that the spatial mode in the gas jet will have the same $33 \mu \mathrm{m}$ spot size, regardless of wavelength.

The gas jet was operated with a constant backing pressure of $\sim 3$ bar of xenon or krypton. The thickness of the gas sample was $0.5 \mathrm{~mm}$, in all cases much shorter than the Rayleigh range of the laser focus. Gas density in the focus was estimated to be $10^{17} \mathrm{~cm}^{-3}$.

Harmonic radiation produced in the gas jet passed into an XUV spectrometer where the beam was spectrally dispersed by a spherical grating (Hitachi 001-0266) and then imaged onto a microchannel plate (MCP) backed by a phosphor screen (Burle APD 3115 32/25/8 I EDR MgF2 P20). The resulting spectrum was recorded with a high dynamic range digital camera. In all cases we averaged the observed spectra over several laser shots and applied corrections for the wavelength-dependent efficiency of the MCP [21] as well as the spectrometer grating [22].

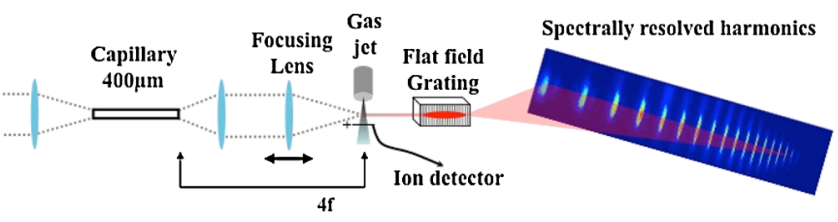

FIG. 1 (color online). Optical layout. Various laser sources were focused into a hollow core waveguide to improve the spatial mode quality and to produce the same beam diameter. The output of the waveguide was imaged into the pulsed valve gas jet in which the high harmonics were produced. The $4 f$ imaging system ensured that the output of the waveguide was imaged into the gas jet.
An ion detector consisting of a wire mesh biased at $-500 \mathrm{~V}$ was located below the pulsed jet to measure the number of ions produced during harmonic generation. To maintain the same focus conditions, the laser beam was focused directly beneath the pulsed jet by finding the lens position that maximized the ion yield. The resulting harmonics propagated as a low divergence beam with no visible annular structure, consistent with short trajectory only.

To verify our detection system, we measured the dependence of the ion signal and the integrated harmonic energy $E(\lambda)$ on pulsed valve gas backing pressure, as shown in Fig. 2. We find that the ion signal increases linearly with backing pressure, confirming that the current is proportional to the number of ions produced in the interaction volume. The total harmonic energy is seen to increase quadratically with pressure. This is to be expected since HHG is a phase-matched process in which the electric fields from individual atoms add coherently [23-25], provided that there is no significant phase mismatch. Calculations of the phase mismatch due to ionization of the sample show that it is negligible with our short $0.5 \mathrm{~mm}$ jet. Reabsorption of the XUV is also calculated to be negligible but in any case is the same for all laser wavelengths for each atom. The observed quadratic increase in harmonic yield with pressure proves that we are operating in a regime that is free of significant phase mismatch or reabsorption. By dividing the harmonic energy by the square of the ion yield, we can remove the measurement's sensitivity to variations in gas pressure, as shown in the lower panel of Fig. 2.

As HHG is a highly nonlinear process, we must be able to accurately control and measure the laser intensity in the
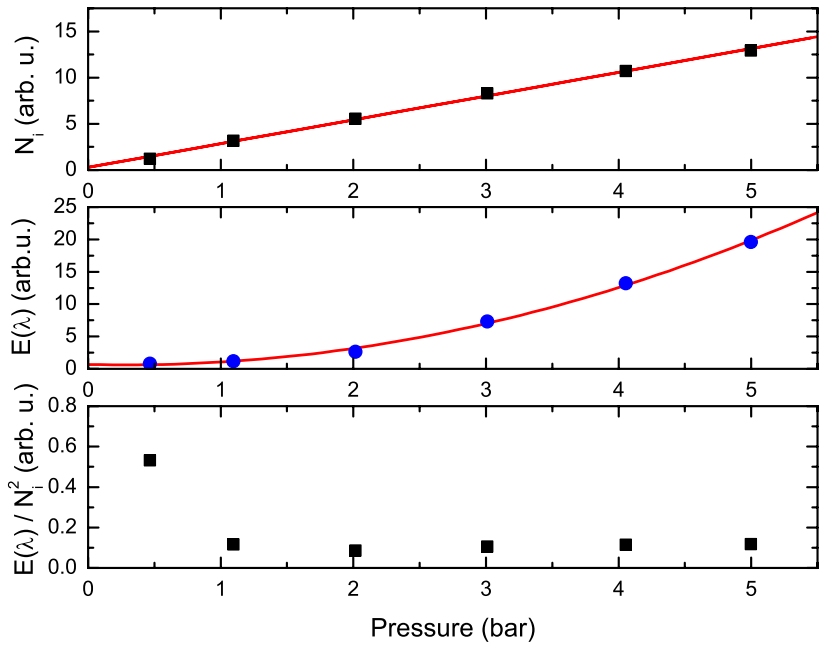

FIG. 2 (color online). Measure ion signal (top) and integrated harmonic yield (middle) from $\mathrm{Xe}$ as a function of the pulsed valve backing pressure. The ion signal is proportional to the backing pressure, and the harmonic yield increases quadratically with pressure, confirming that there is no significant phase mismatch. Dividing the integrated harmonic yield by the square of the ion yield (bottom), we effectively remove the pressure dependence of the measurement. 


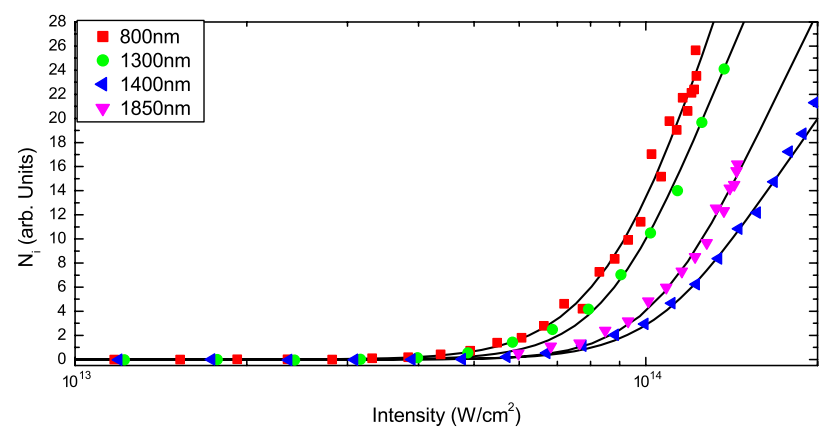

FIG. 3 (color online). Points are the experimentally measured ion signal from Xe recorded for each wavelength and a range of laser pulse energies. The solid lines show the Yudin-Ivanov ionization rate integrated over the focal volume.

interaction volume for all wavelengths. In order to calibrate the peak intensity at each wavelength, the ion yield $N_{i}$ was recorded as a function of pulse energy, as shown by the symbols in Fig. 3. The solid curves were calculated using the Yudin-Ivanov nonadiabatic ionization model [26] integrated over a Gaussian spatial intensity distribution. This model includes both quasistatic tunneling as well as the wavelength-dependent multiphoton contribution to the ionization probability. We minimize the square error between theory and experiment by scaling the horizontal (intensity) axis of the measured data and the vertical (ionization yield) axis of the theoretical curve. This fitting procedure determines the absolute intensity of the laser in the interaction volume for a given pulse energy. The saturation intensity $I_{\text {sat }}$ was determined from the semilog plot of Fig. 3 by extrapolating the asymptotic part of each theoretical curve to zero signal [27]. At saturation, $43 \%$ of the atoms in the most intense region of the focus will have ionized. On such a plot the limiting slope is proportional to the focal volume and gas density. For all wavelengths the limiting slopes agree to within $\pm 32 \%(1 \sigma)$ indicating that the $4 f$ focusing geometry effectively maintains a constant interaction volume.

To facilitate direct measurement of the total XUV energy, the flat field spectrometer was operated without an entrance slit. Because of the beam's small transverse dimension, discrete harmonics are still observed. A representative HHG spectrum is shown in the inset in Fig. 1. By removing the entrance slit, we are able to directly determine the XUV energy by integrating the pixel values from the spectral image on the MCP that correspond to a given range of photon energies. The XUV detection system and this procedure are independent of laser wavelength.

Because of the large range of HHG amplitudes as the laser wavelength is changed, care has to be exercised to maintain linearity of the MCP. To ensure linearity, the MCP voltage was adjusted for each laser condition to ensure that the MCP was not saturating. We separately characterized the voltage-dependent gain of the MCP.

HHG yield as a function of laser intensity was recorded at four wavelengths. We chose two conditions to compare

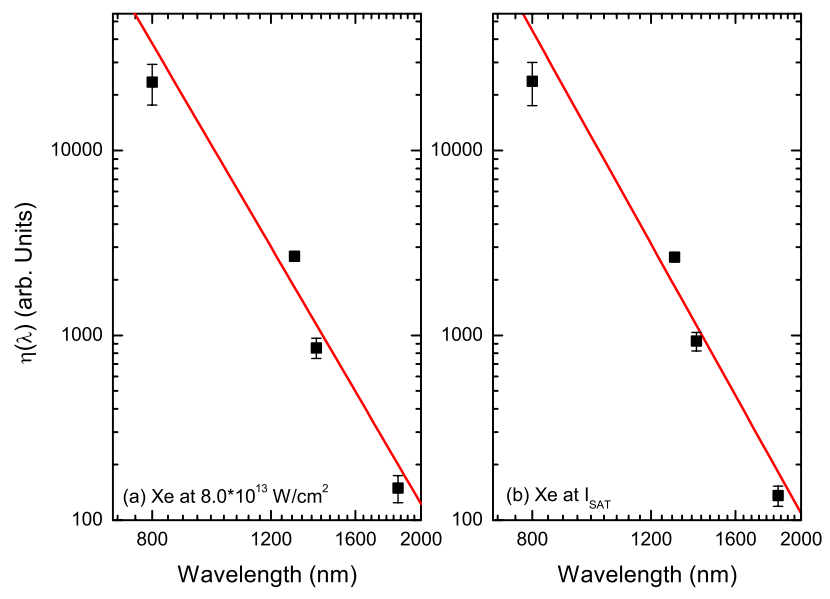

FIG. 4 (color online). Scaling of harmonic yield in Xe in the range 16-32 eV, corrected for the single-atom ionization rate (see text). Results shown at a constant intensity of $8.0 \times$ $10^{13} \mathrm{~W} / \mathrm{cm}^{2}$ (a) and at saturation intensity $I_{\text {sat }}$ (b). The scaling law is $\eta(\lambda) \propto \lambda^{x}$, where $x=-6.3 \pm 1.1$ at $8.0 \times 10^{13} \mathrm{~W} / \mathrm{cm}^{2}$ and $x=-6.6 \pm 1.0$ at $I_{\text {sat }}$.

the HHG efficiency versus wavelength-constant intensity and constant ionized fraction. Figure 4(a) shows the experimental results in $\mathrm{Xe}$ at a constant intensity of $8.0 \times$ $10^{13} \mathrm{~W} / \mathrm{cm}^{2}$. Figure 4(b) shows the experimental results where the ionized fraction was kept constant by setting the laser to the saturation intensity $I_{\text {sat }}$ at each wavelength, as shown in Table I. Experimental results for $\mathrm{Kr}$ gas at a constant intensity of $1.3 \times 10^{14} \mathrm{~W} / \mathrm{cm}^{2}$ are shown in Fig. 5(a) as well as at a constant fraction of the saturation intensity $0.8 I_{\text {sat }}$ in Fig. 5(b).

Our goal is to determine the single-atom scaling, to enable comparison with recent theoretical predictions. To make this comparison, we must convert the coherent emission from $N_{0}$ neutral atoms by dividing $E(\lambda)$ by $N_{0}^{2}$. We directly measure the ion yield $N_{i}=N_{0} P_{\mathrm{YI}}$, and we determine the ionization probability $P_{\mathrm{YI}}$ from the Yudin-Ivanov model fit shown in Fig. 3. We have thus plotted $\eta(\lambda)=$ $E(\lambda) / N_{0}^{2}$ in Figs. 4 and 5. The exponent $x$ for the deduced scaling law $\eta(\lambda) \propto \lambda^{-|x|}$ is shown in Table II.

It was proposed by Yakovlev, Ivanov, and Krausz [28] that it is possible to overcome the single-atom response associated with longer driver wavelengths by reducing the laser intensity. The idea is that you want to produce emission at a particular frequency, and since the cutoff scales as $I \lambda^{2}$, one can reduce $I$. Lower intensity produces fewer

TABLE I. Laser parameters used for the study.

\begin{tabular}{rlrlr}
\hline \hline$\lambda(\mathrm{nm})$ & \multirow{2}{*}{ Source } & $\tau(\mathrm{fs})$ & \multicolumn{2}{c}{$I_{\text {sat }}\left(10^{14} \mathrm{~W} / \mathrm{cm}^{2}\right)$} \\
& & & $\mathrm{Xe}$ & $\mathrm{Kr}$ \\
\hline 800 & Ti:sapph. & 62 & 0.84 & 1.50 \\
1300 & OPA sig. & 130 & 0.84 & 1.44 \\
1400 & OPA sig. & 55 & 1.01 & 1.73 \\
1850 & OPA idl. & 64 & 1.02 & 1.72 \\
\hline \hline
\end{tabular}



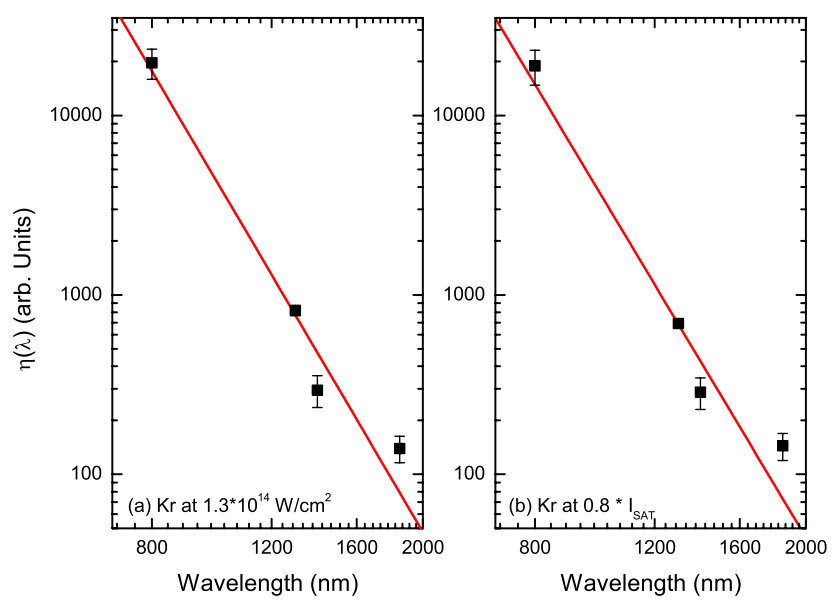

FIG. 5 (color online). Scaling of harmonic yield in $\mathrm{Kr}$ in the range $16-32 \mathrm{eV}$ corrected for the single-atom ionization rate (see text). Results shown are for a constant intensity $1.3 \times$ $10^{14} \mathrm{~W} / \mathrm{cm}^{2}$ (a) as well as at a constant fraction of the saturation intensity $0.8 I_{\text {sat }}$ (b). The scaling law is $\eta(\lambda) \propto \lambda^{x}$, where $x=$ $-6.5 \pm 1.1$ at $1.3 \times 10^{14} \mathrm{~W} / \mathrm{cm}^{2}$ and $x=-6.4 \pm 1.3$ at $0.8 I_{\text {sat }}$.

electrons and thus less phase mismatch. It is not possible for us to test this conjecture, since we intentionally used a very short gas jet to avoid phase mismatch. We also cannot test the scaling at constant $I \lambda^{2}$ because that implies a very low intensity at the longest wavelength, an intensity at which we could not detect any harmonics. Our results suggest just the opposite, that the XUV emission from a short medium in the 16-32 eV range goes down even more rapidly than $\lambda^{-5}$ if the intensity is adjusted as $I(\lambda) \propto \lambda^{-2}$. To achieve Yakovlev's goal, the medium must also be extended or the focusing geometry changed.

In summary, we measured the high harmonic efficiency versus wavelength at constant intensity and at constant ionization fraction, using a short gas jet where phase mismatch is not an issue. By simultaneously measuring the ionization, we normalize to the number of atoms and isolate the single-atom response. We observed a scaling law of $\eta(\lambda) \propto \lambda^{-6.3 \pm 1.1}$ in $\mathrm{Xe}$ and $\lambda^{-6.5 \pm 1.1}$ in $\mathrm{Kr}$ (see Table II). The dramatic decrease in HHG efficiency predicted by Tate et al. [11] came as a surprise to the attosecond community and suggests that there will be significant challenges for experiments employing longer wavelength sources. Our finding that the single-atom HHG

TABLE II. Value of the efficiency scaling exponents obtained for $\eta(\lambda) \propto \lambda^{-|x|}$ integrated in the $16-32 \mathrm{eV}$ range.

\begin{tabular}{lcc}
\hline \hline Gas & Intensity & Exponent \\
\hline $\mathrm{Xe}$ & $8.0 \times 10^{13} \mathrm{~W} / \mathrm{cm}^{2}$ & $-6.3 \pm 1.1$ \\
$\mathrm{Xe}$ & $I_{\text {sat }}$ & $-6.6 \pm 1.0$ \\
$\mathrm{Kr}$ & $1.3 \times 10^{14} \mathrm{~W} / \mathrm{cm}^{2}$ & $-6.5 \pm 1.1$ \\
$\mathrm{Kr}$ & $0.8 I_{\text {sat }}$ & $-6.4 \pm 1.3$ \\
\hline \hline
\end{tabular}

yield decreases even more rapidly than predicted will be an important consideration in the design of future experiments.

The authors thank Thomas Brabec for helpful discussions regarding phase matching and laser technicians François Poitras and Antoine Laramée for their dedicated work on the laser system. We acknowledge funding support from NSERC and AFOSR.

*david.villeneuve@nrc.ca.

[1] P. B. Corkum, Phys. Rev. Lett. 71, 1994 (1993).

[2] K. J. Schafer, B. Yang, L. F. DiMauro, and K. C. Kulander, Phys. Rev. Lett. 70, 1599 (1993).

[3] A. Scrinzi, M. Y. Ivanov, R. Kienberger, and D. M. Villeneuve, J. Phys. B 39, R1 (2006).

[4] P. B. Corkum and F. Krausz, Nature Phys. 3, 381 (2007).

[5] G. Sansone et al., Science 314, 443 (2006).

[6] J. Itatani et al., Nature (London) 432, 867 (2004).

[7] V.-H. Le, A.-T. Le, R.-H. Xie, and C. D. Lin, Phys. Rev. A 76, 013414 (2007).

[8] M. Lewenstein, P. Balcou, M. Y. Ivanov, A. L'Huillier, and P. B. Corkum, Phys. Rev. A 49, 2117 (1994).

[9] B. Sheehy et al., Phys. Rev. Lett. 83, 5270 (1999).

[10] B. Shan and Z. Chang, Phys. Rev. A 65, 011804 (2001).

[11] J. Tate, T. Auguste, H. G. Muller, P. Salières, P. Agostini, and L. F. DiMauro, Phys. Rev. Lett. 98, 013901 (2007).

[12] K. Schiessl, K. L. Ishikawa, E. Persson, and J. Burgdörfer, Phys. Rev. Lett. 99, 253903 (2007).

[13] M. V. Frolov, N. L. Manakov, and A. F. Starace, Phys. Rev. Lett. 100, 173001 (2008).

[14] B. Shan, A. Cavalieri, and Z. Chang, Appl. Phys. B 74, s23 (2002).

[15] T. Popmintchev, M.-C. Chen, O. Cohen, M. E. Grisham, J. J. Rocca, M. M. Murnane, and H. C. Kapteyn, Opt. Lett. 33, 2128 (2008).

[16] E. J. Takahashi, T. Kanai, K. L. Ishikawa, Y. Nabekawa, and K. Midorikawa, Phys. Rev. Lett. 101, 253901 (2008).

[17] Y. Fu et al., Phys. Rev. A 79, 013802 (2009).

[18] G. Doumy, J. Wheeler, C. Roedig, R. Chirla, P. Agostini, and L.F. DiMauro, Phys. Rev. Lett. 102, 093002 (2009).

[19] P. Colosimo et al., Nature Phys. 4, 386 (2008).

[20] H.-C. Bandulet et al., J. Phys. B 41, 245602 (2008).

[21] R. Hemphill, J. Edelstein, and D. Rogers, Appl. Opt. 36, 1421 (1997).

[22] J. Edelstein, M. C. Hettrick, S. Mrowka, P. Jelinsky, and C. Martin, Appl. Opt. 23, 3267 (1984).

[23] P. Balcou, P. Salières, A. L'Huillier, and M. Lewenstein, Phys. Rev. A 55, 3204 (1997).

[24] E. Constant et al., Phys. Rev. Lett. 82, 1668 (1999).

[25] M. B. Gaarde, J. L. Tate, and K. J. Schafer, J. Phys. B 41, 132001 (2008).

[26] G. L. Yudin and M. Y. Ivanov, Phys. Rev. A 64, 013409 (2001).

[27] S. M. Hankin, D. M. Villeneuve, P. B. Corkum, and D. M. Rayner, Phys. Rev. A 64, 013405 (2001).

[28] V.S. Yakovlev, M. Y. Ivanov, and F. Krausz, Opt. Express 15, 15351 (2007). 